\title{
Optimizing QoS for Multimedia Services in Next Generation Network Based on ACO Algorithm
}

\author{
Dac-Nhuong Le \\ Faculty of Information Technology, Haiphong University, Haiphong, Vietnam \\ E-mail: Nhuongld@hus.edu.vn
}

\begin{abstract}
In Next Generation Network (NGN), the backbone of the overall network architecture will be IP network, supporting different access network technologies and types of traffics. NGN will provide advanced services, such as Quality of Service (QoS) guarantees, to users and their applications. Factors affecting the QoS in NGN are speech encoders, delay, jitter, packet loss and echo. The negotiation and dynamic adaptation of QoS is currently considered to be one of the key features of the NGN concept. In this paper, I propose a novel Ant Colony Optimization algorithm to solve model of the optimal QoS for multimedia services in the NGN. Simulation results show that my new approach has achieved near optimal solutions. Comparison of experimental results with a recently researches shows that the proposed algorithm is better performance and it can meets the demand of the optimal QoS for multimedia services in NGN.
\end{abstract}

Index Terms - Quality of Service; Multimedia Services; QoS Matching; Next Generation Networks; Ant Colony Optimization

\section{Introduction}

In Next Generation Network (NGN), the backbone of the overall network architecture will be IP network, supporting different access network technologies such as wireless Local Area Network (WLAN), UMTS Terrestrial Radio Access Network (UTRAN), and WiMax. Moreover, this integrated wireless system, will have to handle diverse types of traffics: data traffics (e.g. web browsing, e-mail, ftp), voice traffic (e.g. voIP), and multimed ia traffics (e.g. video conferencing, online TV, online games), etc... NGN will provide advanced services, such as Quality of Service (QoS) guarantees, to users and their applications. Factors affecting the QoS in NGN are speech encoders, delay, jitter, packet loss and echo. In standardized NGN architecture, at the service level specify requirements for entities at the rim of the network to negotiate and select common codecs for each End to End (E2E) session ${ }^{[1]}$. The $3^{\text {rd }}$ Generation Partnership Project (3GPP) has specified the IP Multimedia Subsystem (IMS) procedures for negotiating multimedia session characteristics between endpoints ${ }^{[2]}$. Under these demanding conditions of media-rich services, network service providers must carefully provision and allocate network resource capabilities to providing personalized and optimal service quality implies more advanced negotiation mechanisms matching user preferences/capabilities against service requirements, network resource capabilities, and operator policy.

Related work on QoS adaptation in IMS networks includes solutions for dynamically adapting multimedia content to fit network/system resource availability. Ozcelebi et al have presented a solution to enhancing E2E QoS for multimedia streaming in $\mathrm{IMS}^{[3]}$. Boula et al introduced an enhanced IMS architecture featuring cross-layer monitoring and adaptation mechanisms to maximizing perceived QoS ${ }^{[4]}$. The DAIDALOS ${ }^{[5]}$ and ENTHRONE $^{[6]}$ projects is further research on multimedia session control and content adaptation. In additional related work, we can be found on profile matching ${ }^{[7]}$, decision-making for the optimization of service parameters ${ }^{[8-9]}$, and the description of various service and transport configurations for service negotiation ${ }^{[10-12]}$, limited solutions unite these aspects to support delivery of personalized multimed ia services in the NGN. Khan has proposed an heuristic algorithm for finding near optimal solutions for cases with a large number of variables to solve the quality adaptation in a multisession multimedia system proble ${ }^{[13]}$. A different approach to formulating the optimization problem may be to use tools specified as part of the MPEG DIA standard, Usage Environment Description tools, Universal Constraints Description tool, and Adaptation QoS tools has presented by Mukherjee et $\mathrm{al}^{[14]}$. In [15], the authors have proposed the high-level concept of a QoS Matching and Optimization Function (Q-MOF) to be included along the E2E signaling path in the service control layer of the NGN architecture and heuristic algorithm for finding near optimal solutions.

In this paper, I propose a novel Ant Colony Optimization (ACO) algorithm to solve the Optimal QoS for Multimedia Services (OQMS) problem. Simulation results show that my new approach has achieved near optimal solutions. I have compared to evaluate the effectiveness of the proposed algorithm with the recently researches. The rest of this paper is organized as follows. Section 2 and section 3 present QMOF model in the NGN architecture ${ }^{[15]}$ and OQMS problem formulation. Section 4 presents our new 
algorith m for OQMS problem based on ACO algorithm. Section 5 presents our simulation and analysis results, and finally, section 6 concludes the paper.

\section{Q-MOF in the NGN Architecture}

Fig. 1 presents the ITU-T NGN release an architecture based on the concept of independence between the transport stratum and service stratum ${ }^{[1]}$. In the service stratum, service control functions are based on an IMS service environment and support the provisioning of real-time Session Initiation Protocol (SIP) based multimedia services. The application support functions and service support functions can impact sessions on behalf of services. During session negotiation, QoS requirements are extracted by service control functions and used to is sue resource reservation and authorization requests to the Resource Admission Control Functional architecture (RACF). In order to provide enhanced QoS control mechanis ms, Q-MOF have introduced in the service stratum as a new application server. The QMOF is designed to be included along the E2E signaling path, providing support for advanced QoS parameter matching and optimization log ic within the
QoS negotiation process. Actual service adaptation may be performed at communication endpoints or in the network. While today's Internet model is based on a user-centric view of the network, the IMS model uses a more operator-centric approach ${ }^{[15]}$.

In an actual network implementation, the authors in [15] proposed the Q-MOF to be included in a service provider domain as a generic reusable functionality to providing a better service to users, introducing enhanced QoS support in the network as a reusable service capability would benefit both the service provider and third-party service/application providers. The service provider would have additional means to control, differentiate, and appropriately charge the QoS a particular user receives for a given mu ltimedia service. Third-party service/application providers would have to specify a service profile stating service requirements and options and would further be relieved from implementing complex QoS decision making functionality for each new introduced service, hence leading to simplified provisioning and possibly quicker time-to-market for new services requiring such mechanis ms. A multimedia service is composed of two or more media components such as audio flows, video flows, graphics data, etc... ${ }^{[15]}$

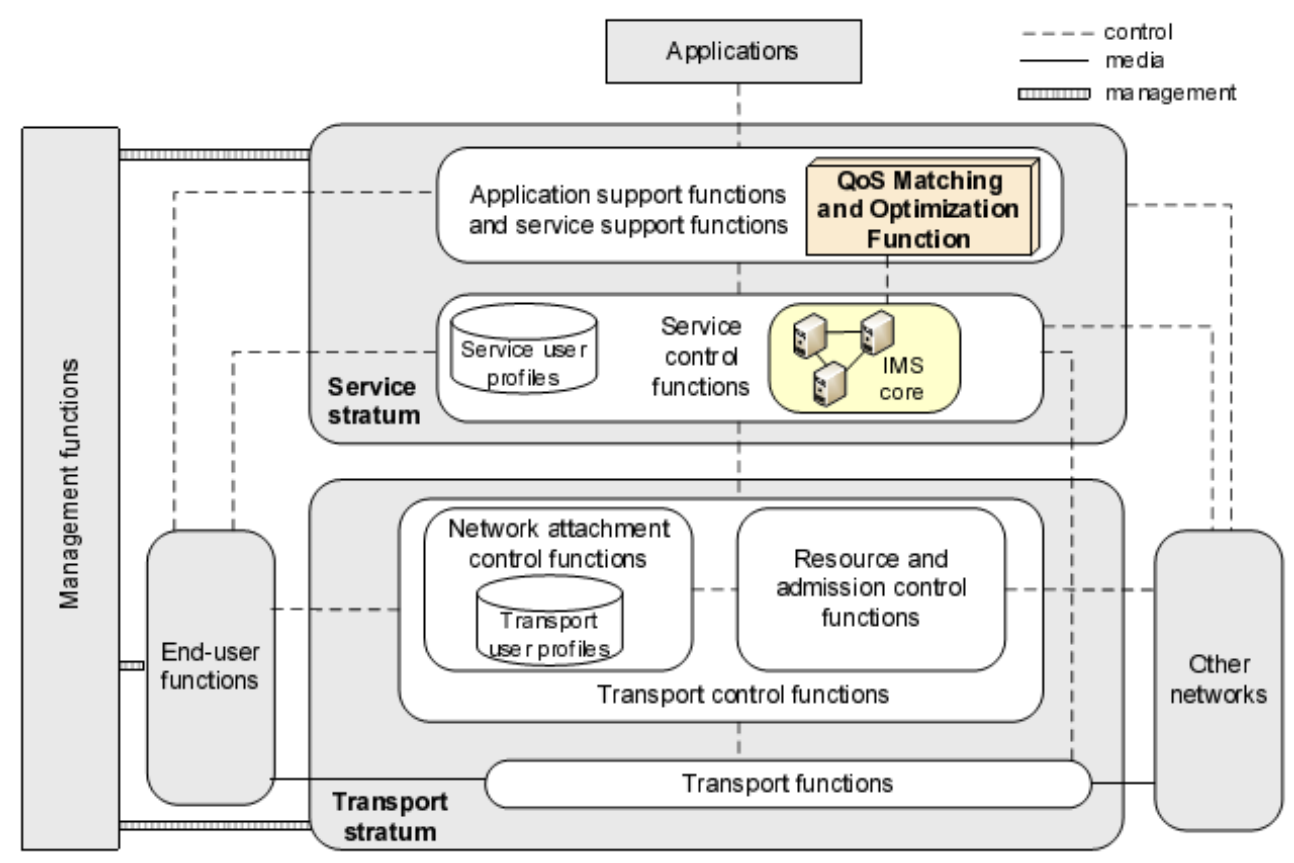

Fig. 1: Q-MOF in the NGN architecture

I assume that a multimedia service exists in one or more versions to meet heterogeneous user and network capabilities. We specify service versions as differing in the included media components. Each med ia component may be configured by choosing from offered alternative operating parameters (e.g., different codecs, frame rates, resolutions, etc.). We refer to the overall service configuration as the set of chosen operating parameters for all included media components ${ }^{[13,15]}$.

\section{Model of OQMS Problem}

The optimal QoS for multimedia services (OQMS) problem can be formulated as a multi-choice multidimension 0-1 knapsack problem (MMKP), for which I introduce the following notation:

- $F=\left(f_{1}, \ldots, f_{n}\right)$ is set of different media flows ( $n$ is number of different media flows), of which flows 
$f_{1}, \ldots, f_{h}$ are in the downlink direction and flows $f_{h+1}, \ldots, f_{n}$ are in the uplink direction.

- The flow $f_{i}$ has $p_{i}$ operating points.

- $r_{i j}=\left(r_{i j l}, \ldots, r_{i j q}, \ldots, r_{i j m}\right)$ is set of required resources $r$ for operating point $p_{j}$ and media flow $f_{i}$, of which $m$ is number of resources, $r_{i j 1}, \ldots, r_{i j q}$ are corresponding to bandwidth assigned within different QoS classes (differing in delay, loss, jitter, and bandwidth guarantees). For a single operating point, we assume only one of the values $r_{i j 1}, \ldots, r_{i j q}$ to be greater than zero, while all others are equal to zero. This is because only one QoS class is chosen per operating point for a media flow.

- $B_{\text {dounlink }}$ and $B_{\text {uplink }}$ are maximum available downlink and uplink bandwidth determined by user terminal capabilities and access network. They represent the resource constraints for $r_{i j 1}, \ldots, r_{i j q}$.

- $R=\left(R_{q+1}, \ldots, R_{m}\right)$ is the consumption of a particular resource across all media flows (e.g., cost for all flows must be less than a specified amount).

- $R_{i}=\left(R_{i 1}, \ldots, R_{i m}\right)$ is regarding resource consumption per flow $f_{i}$ (e.g., cost for a media flow must be less than a specified amount).

- $u_{i}\left(r_{i j}\right)$ is the utility value for operating point $p_{j}$ and media flow $f_{i}$.

- $w_{i}$ is weight factors assigned to utility values to indicate the relative importance of media flows.

- $x_{i j}=\left\{\begin{array}{l}1 \text { if operating point } p_{j} \text { is chosen per media flow } f_{i} \\ 0 \text { otherwise }\end{array}\right.$

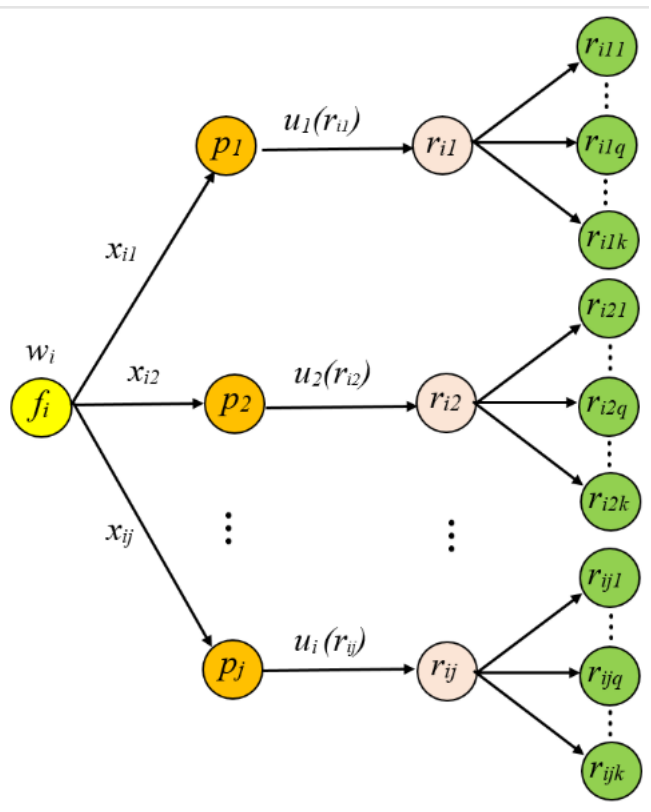

Fig. 2: Flow $i^{\text {th }}$ in OQMMS problem
Then the formulation OQMS for multi-flow resource constrained utility maximization is:

$$
\max \sum_{i=1}^{n} \sum_{j=1}^{p_{i}} w_{i} x_{i j} u_{i}\left(r_{i j}\right)
$$

Subject to

$$
\begin{gathered}
\sum_{i=1}^{h} \sum_{j=1}^{p_{i}} \sum_{k=1}^{q} x_{i j} r_{i j k} \leq B_{\text {downlink }} \\
\sum_{i=h+1}^{n} \sum_{j=1}^{p_{i}} \sum_{k=1}^{q} x_{i j} r_{i j k} \leq B_{\text {uplink }} \\
\sum_{i=1}^{n} \sum_{j=1}^{p_{i}} x_{i j} r_{i j k} \leq R_{k}, \forall k=\overline{q+1, . . m} \\
\sum_{j=1}^{p_{i}} x_{i j} r_{i j k} \leq R_{i k}, \forall k=\overline{1 . . m, \forall i=\overline{1 . . n}} \\
\sum_{i=1}^{n} \sum_{j=1}^{p_{i}} x_{i j}=1, x_{i j} \in\{0,1\}, \forall i=\overline{1 . . n}, \forall j=\overline{1 . . p_{i}}
\end{gathered}
$$

\section{ACO based Algorithm for the OQMS}

\subsection{Ant Colony Optimization}

The ACO algorithm is originated from ant behavior in the food searching. When an ant travels through paths, from nest food location, it drops pheromone. According to the pheromone concentration the other ants choose appropriate path. The paths with the greatest pheromone concentration are the shortest ways to the food. The optimization algorithm can be developed from such ant behavior. The first ACO algorithm was the Ant System ${ }^{[16]}$, and after then, other implementations of the algorithm have been developed $^{[17-18]}$.

There are some ACO based solutions for a more restricted variant of Knapsack Problem (KP), namely Multi-choice Knapsack Problem (MKP) and Multichoice Multi-dimension Knapsack Problem (MMKP) when MKP resources have multiple dimensions. However there is no concept of group in MKP. As a result, MKP can be thought of as a restricted version of MMKP, which has all objects in a single group ${ }^{[19-22]}$.

The exist algorithms differ in deciding which component of the problem should be regarded as the pheromone depositing component and in the mechanisms of pheromone updating:

- Pheromone Trails on Each Object: The first way is to lay pheromone trails on each object belonging to the current solution set the amount of pheromone represents the preference of the object ${ }^{[19]}$. 
- Pheromone Trails on Each Pair: In this case, pheromone trails are laid on each pair $\left(o_{i}, o_{\mathrm{j}}\right)$ of successively selected objects of the solution set the idea is to increase the desirability of choosing object $o_{\mathrm{j}}$ when the last selected object is $o_{i}{ }^{[20]}$.

- Pheromone Trails on All Pair: The third one is to lay pheromone trails on all pairs of different objects of the solution set. Here, the idea is to increase the desirability of choosing simultaneously two objects of $S^{[21]}$.

- Pheromone Diffusion Model: The forth approach follows the same principle as the first one. Additionally it uses a pheromone diffusion scheme where pheromone trails are laid on objects that tend to occur together in previous solutions ${ }^{[22]}$.

\subsection{Solving the OQMS Based on ACO}

In this section, I present application of ACO technique with the dynamic local heuristic information for the OQMS problem. My new algorith $\mathrm{m}$ is described as follows:

Let $\tau_{\min }$ and $\tau_{\max }$ are lower and upper bounds on pheromone values and pheromone $\tau_{\min }<\tau<\tau_{\max }$. I initialize all pheromone trails to $\tau_{\max }$. The algorithm chooses first group $g_{i}$ has been randomly chosen. For each candidate group $o_{j}$, the pheromone factor $\tau_{S_{k}}\left(g_{j}\right)$ is initialized to $\tau\left(o_{i}, o_{j}\right)$. Each time a new group $g_{l}$ is added to the solution $S_{k}$, for each candidate group $g_{j}$, the pheromone factor $\tau_{S_{k}}\left(g_{j}\right)$ is incremented by $\tau\left(o_{l}, o_{j}\right)$. And the highest pheromone factor can be defined as

$$
\tau_{S_{k}}\left(g_{i}\right)=\sum_{g_{j} \in S_{k}} \tau\left(o_{i}, o_{j}\right)
$$

When the algorithm starts, ants can perform search in order of group that impose the lowest restriction on the next choices. The key point is to decide which components of the constructed solutions should be rewarded, and how to exploit these rewards when constructing new solutions.

A solution is a set of selected objects $S=\left\{o_{i j} \mid x_{o_{i j}}=1\right\}$. Say $x_{o_{i j}}=1$ is the mean an object $o_{i j}$ is selected and the corresponding decision variable $x_{i j}$ has been set to 1 . We must constructed solutions $S=\left\{o_{i_{1} j_{1}}, \ldots, o_{i_{n} j_{n}}\right\}$, pheromone trails are laid on each objects selected in $S$. So pheromone trail $\tau_{i j}$ will be associated with object $o_{i j . .}$ Fig. 3 show an example of the solution $S$ in my algorithm.

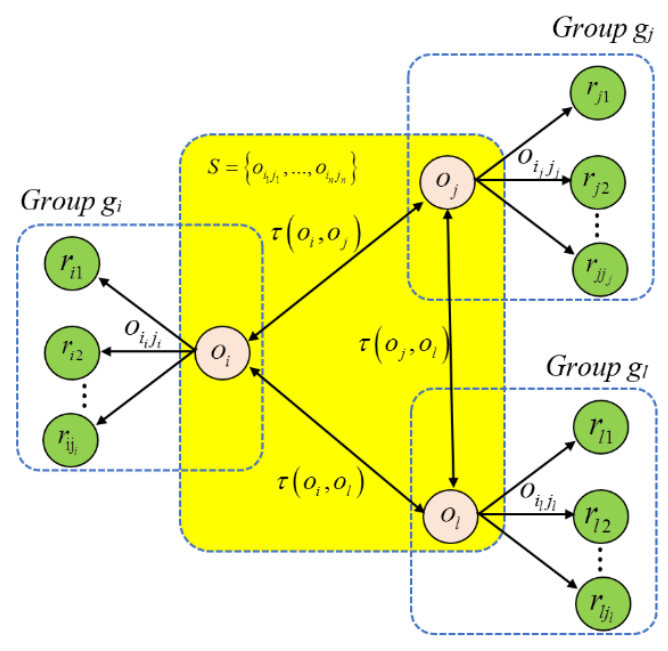

Fig. 3: Solution $\mathrm{S}$ in our algorithm

Let $S_{k}$ be the set of the selected objects at the $k^{\text {th }}$ Iteration. The heuristic factor $s_{k}\left(O_{i j}\right)$ is determined by:

$$
s_{k}\left(O_{i j}\right)=\frac{w_{i} u_{i}\left(r_{i j}\right)}{\sum_{i=1}^{m} \frac{r_{i j l}}{d_{S_{k}}(l)}}
$$

where,

- $d_{S_{k}}(l)=R_{l}-\sum_{o_{i j} \in S_{k}} r_{i j l}$

- $l$ is index of resource $R$ across all media flows.

- $S_{k}$ is the dynamic heuristic information will be changed from step to step.

My algorithm select groups in the same way as it selects the objects. I have unique idea of a separate pheromone trail for groups to save the ordering of groups that lead to a good solution. The group pheromone trail also follow a min-max ant system approach and initialized to the max pheromone value ${ }^{[24]}$.

Once each ant has constructed a solution, pheromone trails laying on the solution objects are updated according to the ACO meta-heuristic. First, all amounts are decreased in order to simulate evaporation. This is done by multiplying the quantity of pheromone laying on each object by a pheromone persistence rate $(1-P)$ such that $0 \leq P \leq 1$. Then, pheromone is increased for all the objects in the best solution of the iteration. When constructing a solution, an ant starts with an empty knapsack. At the first construction step an ant selects a group randomly and at all the latter steps, groups are selected according to their as sociated pheromone value.

After selecting a group, the algorithm removes all the bad Candidates that violates resource constraints. It then updates the local heuristic information of the remaining candidate objects of the group and selects an object according to the following probability equation: 


$$
P_{S_{k}\left(o_{i j}\right)}=\frac{\left[\tau_{S_{k}}\left(O_{i j}\right)\right]^{\alpha}\left[\eta_{S_{k}}\left(O_{i j}\right)\right]^{\beta}}{\sum_{O_{i j} \in \text { Candidates }}\left[\tau_{S_{k}}\left(O_{i j}\right)\right]^{\alpha}\left[\eta_{S_{k}}\left(O_{i j}\right)\right]^{\beta}}
$$

in which,

- Candidates are all items from the currently selected group which do not violate any resource constraints.

- $\tau_{S_{k}}$ is the pheromone factor of the dynamic heuristic information $S_{k}$ and computed by equation (8)

- $\eta_{S_{k}}$ is the desirability of $S_{k}$.

- The influence of the pheromone concentration to the probability value is presented by the constant $\alpha$, while constant $\beta$ do the same for the desirability. Parameters $\alpha$ and $\beta$ control the relative importance of pheromone trail versus local heuristic value.

More precisely, let $S_{\text {internalbest }}$ be the best solution constructed during the current cycle. The quantity of pheromone increased for each object is defined by:

$$
G\left(S_{\text {internalbest }}\right)=\frac{1}{\sum_{k=1}^{n} P_{S_{k}\left(o_{i j}\right)}} \times \sum_{o_{i j} \in S_{\text {intermalbest }}} w_{i} u_{i}\left(r_{i j}\right)
$$

After each iteration, group pheromone values have much better than the original pheromone trail for ants. The best solution updates the group pheromone trail. All the adjacent groups get the highest amount of pheromone value that gradually diminishes as the distance between groups increases.

The Candidategroups data structure maintains a list of feasible candidate groups which can be considered next. After each ant has constructed a solution, the best solution of that iteration is identified and a random local search procedure and a random item swap procedure is applied to improve it. Then pheromone trail is updated according to the best solution. Also it maintains a database of top $k$ solutions. After each iteration a small amount of pheromone is deposited in the pheromone trails of the objects belonging to the top $k$ solutions. The motivation behind this strategy is to ensure quick convergence on good solutions and to explo re better areas more thoroughly. The algorith $m$ stops either when an ant has found an optimal solution (when the optimal bound is known), or when a maximum number of cycles has been performed.

Our algorith m based on ACO for OQMS described as follows:

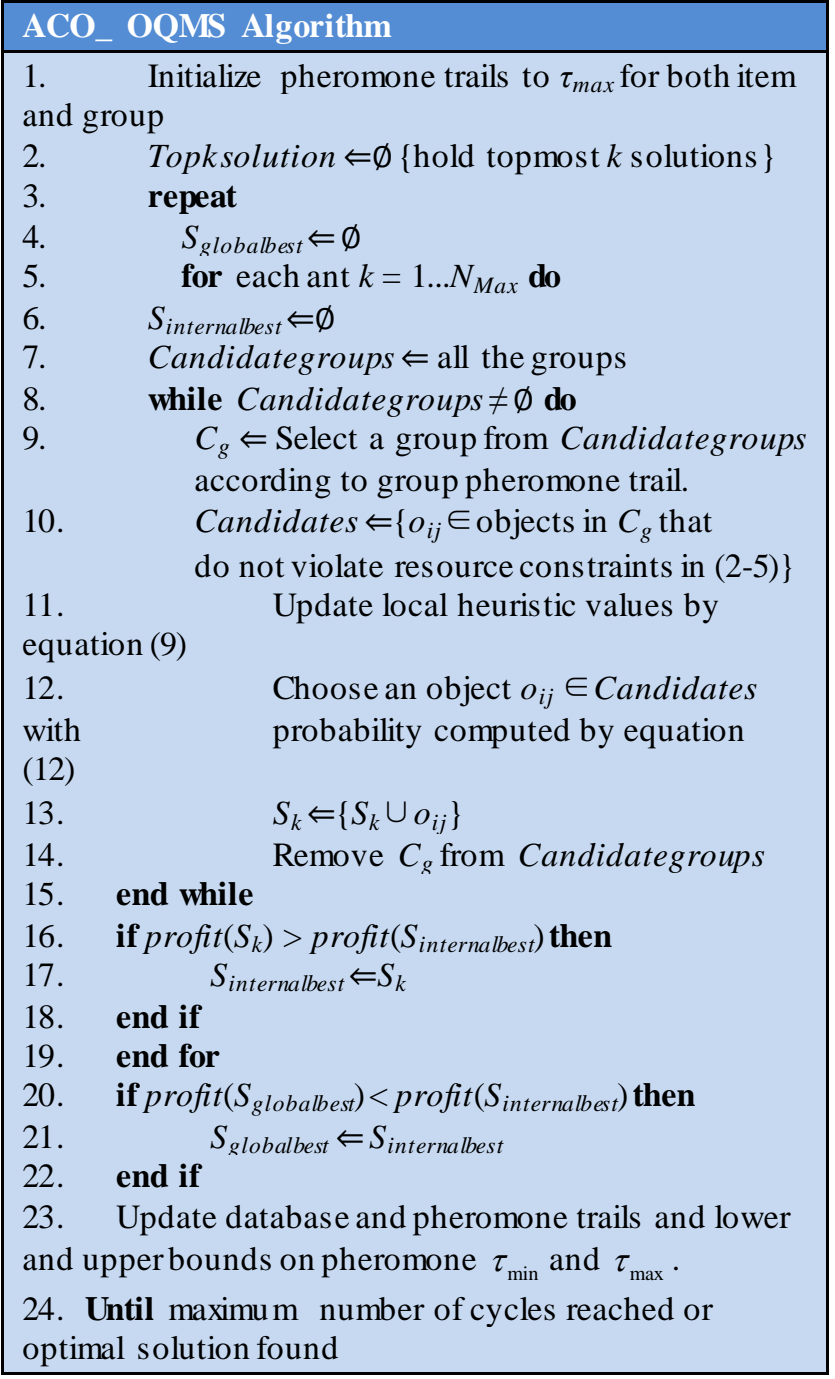

\section{Experiments and Results}

In the first experiment, I implemented the similar prototype Audio/Video Call (A VC) service allow two end users to engage in an audio/video call in a laboratory IMS testbed ${ }^{[15,25]}$. The AVC service matching parameter and service requirement with conversational audio and video is shown in table 1 . We assume the uplink direction from the call initiating user A to the terminating user B. The user capabilities and operator constraints is shown in table 2.

For illustration, I assume a specification of constraints follow:

- Set of different media flows:

$$
F=\left(f_{1}, f_{2}, f_{3}, f_{4}\right) \text {. }
$$

- Flows $f_{1}, f_{2}$ are audio downlink and video downlink and flows $f_{3}, f_{4}$ are audio uplink and video uplink.

- Operating points:

$$
P=\left(p_{1}, p_{2}, p_{3}, p_{4}\right)=(3,8,3,8)
$$


- Utility value $u_{i}\left(r_{i j}\right)$ in the range of $[0,1]$

- $B_{\text {downlink }}=1200 \mathrm{kbps}$

- $B_{\text {uplink }}=800 \mathrm{kbps}$

- A hypotheticalprice: 10 [monetary unit/bit].

- Maximum cost 20000 [monetary unit/s]

- Cost as bandwidth class $q$ [bit/s] x price class $q$ [monetary unit/b].

Our operating parameters, resource vectors and corresponding utilities are specified in the service profile and summarized in Table 3.

The parameters of our algorithm are set as follows:

- $\mathrm{N}_{\mathrm{MAX}}=50, k=10, \alpha=1, \beta=5$

- $P=0.01, \tau_{\text {min }}=0.01, \tau_{\text {max }}=8$

- Pheromone deposited $=0.1$

- Diffusion rate $=0.5$

- Maximum number of cycles: 500
The results of objective value and resource configuration of our algorithm updated step by step show in table 4 . The experimental results with the similar input data sets in [15] show that resource configurations of our algorithm is similar results solved by GLPK (GNU Linear Programming Kit). So, this is a promising approach to solve this problem.

In the second experiment, we generated random input data to verify the effectiveness of our algorith m follow constraints below. The experiment was conducted on Genuine Intel囚 CPU DuoCore $3.0 \mathrm{GHz}, 2 \mathrm{~GB}$ of RAM machine. I ran experiment our ACO algorithm. The problems tackled, object value, and time processing shown in table 5, fig. 4 and fig.5.

\section{Conclusions}

In this paper, I propose a novel Ant Colony Optimization algorithm to solve OQMS problem. Simulation results show that our new approach has achieved near optimal solutions. The effectiveness and the efficiency of our algorithm by comparing with the recent researches and it can meets the demand of QoS matching for multimedia services in NGN. Optimizing QoS negotiation models in cases involving multiple providers in service delivery is our next research goal.

Table 1: Matching parameter set for AVC service ${ }^{[15]}$

\begin{tabular}{|c|c|c|}
\hline \multicolumn{2}{|c|}{ AVC service matching parameter set } & $\begin{array}{c}\text { Service requirements } \\
\text { audio, video }\end{array}$ \\
\hline$p_{1}$ & Media Component & audio: mpeg, gsm. video: mpeg, h26 \\
\hline$p_{2}$ & Codecs & 46 \\
\hline$p_{3}$ & Min Bandwidth Downlink & 150 \\
\hline$p_{4}$ & Max Delay Downlink & 10 \\
\hline$p_{5}$ & Max Jitter Downlink & 1 \\
\hline$p_{6}$ & Max Loss Downlink & 46 \\
\hline$p_{7}$ & Min Bandwidth Uplink & 150 \\
\hline$p_{8}$ & Max Delay Uplink & 10 \\
\hline$p_{9}$ & Max Jitter Uplink & 1 \\
\hline$p_{10}$ & Max Loss Uplink & $176 \times 144$ \\
\hline$p_{11}$ & Resolution Local & $176 \times 144$ \\
\hline$p_{12}$ & Resolution Remote & \\
\hline
\end{tabular}

Table 2: Matching parameter set for AVC service ${ }^{[15]}$

\begin{tabular}{|c|c|c|c|}
\hline Parameters & User A capabilities & User B capabilities & Operator constraints \\
\hline$p_{1}$ & $\begin{array}{l}\text { audio, video, data, image, } \\
\text { model }\end{array}$ & $\begin{array}{c}\text { audio, video, data, image, } \\
\text { model }\end{array}$ & $\begin{array}{c}\text { User A and B: audio, video, } \\
\text { data, image, text }\end{array}$ \\
\hline$p_{2}$ & $\begin{array}{l}\text { audio: peg, pcm, gsm; } \\
\text { video: mpeg, mjpeg, h263 }\end{array}$ & $\begin{array}{c}\text { audio: peg, pcm, gsm; } \\
\text { video: mpeg, mjpeg, h263 }\end{array}$ & $\begin{array}{c}\text { Not Allowed : } \\
\text { audio, G729, audio G723 }\end{array}$ \\
\hline$p_{3}$ & 1200 & 1300 & 1400 \\
\hline$p_{4}$ & 150 & 150 & N/A \\
\hline$p_{5}$ & N/A & N/A & N/A \\
\hline$p_{6}$ & N/A & N/A & N/A \\
\hline$p_{7}$ & 800 & 1000 & 1400 \\
\hline$p_{8}$ & 150 & 150 & N/A \\
\hline$p_{9}$ & N/A & N/A & N/A \\
\hline$p_{10}$ & N/A & N/A & N/A \\
\hline$p_{11}$ & $1204 \times 768$ & N/A & N/A \\
\hline$p_{12}$ & N/A & $1204 \times 768$ & N/A \\
\hline
\end{tabular}


Table 3: Operating parameters, resource vect ors and utilities for AVC flows ${ }^{[15]}$

\begin{tabular}{|c|c|c|c|c|c|}
\hline$\overline{\text { Flow }}$ & O pe rating parameter & Resource vectors & Bandwidth [kpbs] $r_{i j 1}$ & Cost $r_{i j 1}$ & Utility value $u_{i}\left(r_{i j}\right)$ \\
\hline \multirow{3}{*}{$f_{i}$} & $p_{1}$ & $r_{11}$ & 21 & 210 & 0.5 \\
\hline & $p_{2}$ & $r_{12}$ & 34 & 340 & 0.8 \\
\hline & $p_{3}$ & $r_{13}$ & 64 & 640 & 1.0 \\
\hline \multirow{8}{*}{$f_{2}$} & $p_{1}$ & $r_{21}$ & 25 & 900 & 0.2 \\
\hline & $p_{2}$ & $r_{22}$ & 90 & 900 & 0.4 \\
\hline & $p_{3}$ & $r_{23}$ & 370 & 3700 & 0.5 \\
\hline & $p_{4}$ & $r_{24}$ & 400 & 4000 & 0.6 \\
\hline & $p_{5}$ & $r_{25}$ & 781 & 7810 & 0.7 \\
\hline & $p_{6}$ & $r_{26}$ & 1015 & 10150 & 0.8 \\
\hline & $p_{7}$ & $r_{27}$ & 1400 & 14000 & 0.9 \\
\hline & $p_{8}$ & $r_{28}$ & 2000 & 20000 & 1.0 \\
\hline \multirow{3}{*}{ f3 } & $p_{1}$ & $r_{31}$ & 21 & 210 & 0.5 \\
\hline & $p_{2}$ & $r_{32}$ & 34 & 340 & 0.8 \\
\hline & $p_{3}$ & $r_{33}$ & 64 & 640 & 1.0 \\
\hline \multirow{8}{*}{$f_{4}$} & $p_{1}$ & $r_{41}$ & 25 & 900 & 0.2 \\
\hline & $p_{2}$ & $r_{42}$ & 90 & 900 & 0.4 \\
\hline & $p_{3}$ & $r_{43}$ & 370 & 3700 & 0.5 \\
\hline & $p_{4}$ & $r_{44}$ & 400 & 4000 & 0.6 \\
\hline & $p_{5}$ & $r_{45}$ & 781 & 7810 & 0.7 \\
\hline & $p_{6}$ & $r_{46}$ & 1015 & 10150 & 0.8 \\
\hline & $p_{7}$ & $r_{47}$ & 1400 & 14000 & 0.9 \\
\hline & $p_{8}$ & $r_{48}$ & 2000 & 20000 & 1.0 \\
\hline
\end{tabular}

Table 4: Resource configurations for the ACO_ OQMS Algorithm updated step by step

\begin{tabular}{|c|c|c|}
\hline Number of cycles & Resource Configuration & Objective value \\
\hline $\mathbf{9 3}$ & $\left(r_{13}, r_{23}, r_{33}, r_{44}\right)$ & 2.770 \\
\hline $\mathbf{1 2 8}$ & $\left(r_{13}, r_{25}, r_{33}, r_{43}\right)$ & 2.839 \\
\hline $\mathbf{1 8 7}$ & $\left(r_{13}, r_{26}, r_{33}, r_{42}\right)$ & 2.840 \\
\hline $\mathbf{2 6 4}$ & $\left(r_{13}, r_{24}, r_{33}, r_{44}\right)$ & 2.840 \\
\hline $\mathbf{3 3 1}$ & $\left(r_{13}, r_{25}, r_{33}, r_{44}\right)$ & 2.909 \\
\hline $\mathbf{3 7 5}$ & $\left(r_{13}, r_{26}, r_{33}, r_{43}\right)$ & 2.909 \\
\hline $\mathbf{4 1 7}$ & $\left(r_{13}, r_{26}, r_{33}, r_{44}\right)$ & $\mathbf{2 . 9 8 0}$ (Optimal) \\
\hline
\end{tabular}

Table 5: The problems tackled and results of ACO_OQMS Algorithm

\begin{tabular}{|c|c|c|c|}
\hline \multirow{2}{*}{ Problems } & \multirow{2}{*}{ No. of flows n } & \multicolumn{2}{|c|}{ Operating parameter } \\
\cline { 3 - 4 } & 2 & Downlink $\left(p_{1}, \ldots, p_{h}\right)$ & Uplink $\left(p_{h+1}, \ldots, p_{n}\right)$ \\
\hline$\# 1$ & 2 & $(2)$ & $(2)$ \\
\hline$\# 2$ & 4 & $(3)$ & $(4)$ \\
\hline$\# 3$ & 4 & $(4)$ & $(3,8)$ \\
\hline$\# 4$ & 4 & $(3,7,4)$ & $(9)$ \\
\hline$\# 5$ & 6 & $(6,8,9)$ & $(8,5,4)$ \\
\hline$\# 6$ & 6 & $(10,15)$ & $(8,10,12,13)$ \\
\hline$\# 7$ & 8 & $(10,15,20,25)$ & $(20,10,30,25,15)$ \\
\hline$\# 8$ & 8 & $(15,20,30)$ & $(40,35,50)$ \\
\hline$\# 9$ & 8 & $(20,25,30,35,45)$ & \\
\hline$\# 10$ & & & \\
\hline
\end{tabular}




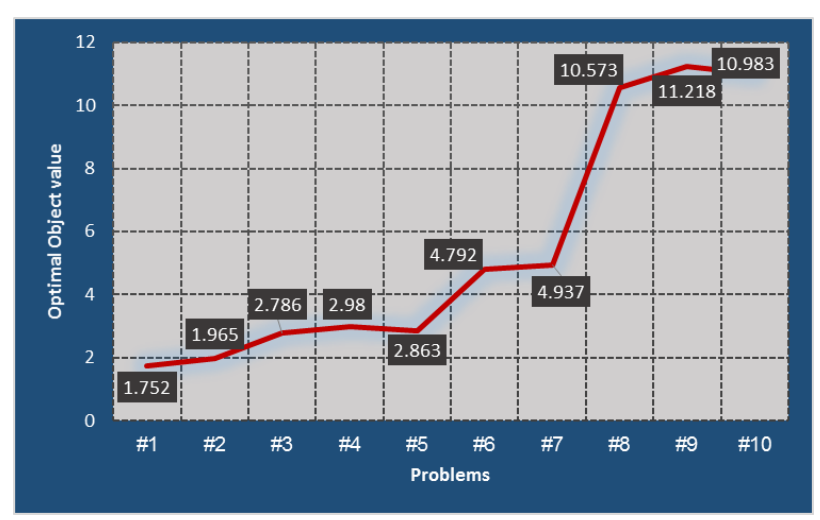

Fig. 4: Problems tackled's optimal object values

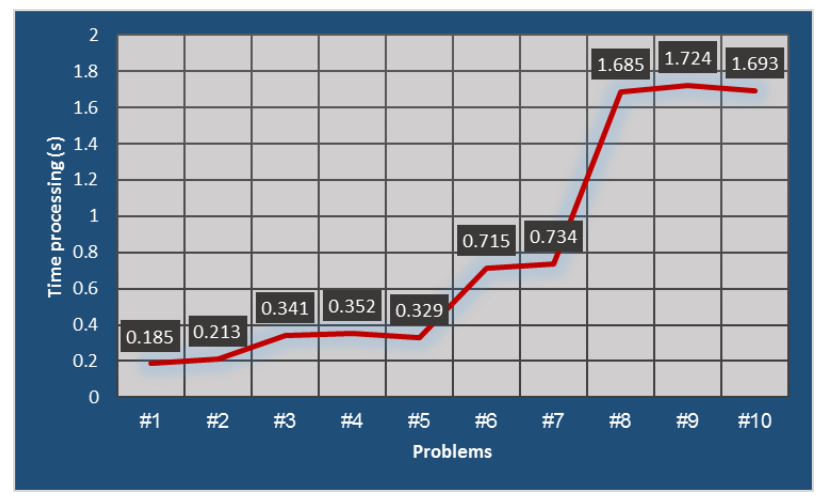

Fig. 5: Comparing times processing

\section{References}

[1] ITU-T Recommendation Y.2012. Functional requirements and architecture of the $N G N$ release 1,2006

[2] 3GPP TS 23.228: IP Multimedia Subsystem (IMS); Stage 2, Release 82008

[3] Ozcelebi, T., Radovanovic, R., Chaudron, M.: Enhancing End-to-End QoS for Multimedia Streaming in IMS-Based Networks. In: ICSNC, pp. 48-53, 2007.

[4] Boula, L., Koumaras, H., Kourtis, A.: An Enhanced IMS Architecture Featuring CrossLayer Monitoring and Adaptation Mechanisms. ICAS, Spain (2009)

[5] IST DAIDALOS - EU FP6,http://www.istdaidalos.org/

[6] IST ENTHRONE - EU FP6,http://www.istenthrone.org/

[7] Houssos, N., et al.: Advanced Adaptability and Profile Management Framework for the Support of Flexible Service Provision. IEEE Wireless Communications, pp.52-61, 2003.
[8] Khan, S.: Quality Adaptation in a Multisession Multimedia System: Model, Algorithms and Architecture.PhD Thesis, Univ. of Victoria, 1998

[9] Wang, Y., Kim, J.-G., Chang, S.-F., Kim, H.-M.: Utility-Based Video Adaptation for Universal Multimedia Access (UMA) and Content-Based Utility Function Prediction for Real-Time Video Transcoding. IEEE Trans. on Multimedia 9(2), pp.213-220, 2007

[10] Guenkova-Luy, T., Kassler, A.J., Mandato, D.: End-to-End Quality-of-Service Coordination for Mobile Multimedia Applications. IEEE J. Selec. Areas Com-mun. 22(5), pp.889-903, 2004

[11] Rosenberg, J., et al.: SIP: Session Initiation Protocol. IETF RFC 32612002.

[12] Handley, H., Jacobson, V.: SDP: Session Description Protocol. IETF RFC 2327, 1998.

[13] Khan, S.: Quality Adaptation in a Multisession Multimedia System: Model, Algorithms and Architecture. PhD Thesis, Univ. of Victoria, 1998.

[14] Mukherjee, D., Delfosse, E., Kim, J.-G., Wang, Y.: Optimal Adaptation Decision-Taking for Terminal and Network Quality of Service. IEEE Trans. on Multime-dia 7(3), pp.454-462, 2005

[15] Lea Skorin-Kapov, and Maja Matijasevic, Modeling of a QoS Matching and Optimization Function for Multimedia Services in the NGN, MMNS 2009, LNCS 5842, pp.55-68, 2009.

[16] M. Dorigo, V. Maniezzo, and A. Colorni, Ant system: Optimization by a colony of cooperating agents, IEEE Trans. on System, MAN, and Cybernetics-Part B, vol. 26, pp. 29-41, 1996.

[17] E. Rajo-Ig lesias, O. Quevedo-Teruel, Linear Array Synthesis using an Ant Colony Optimization based Algorithm, IEEE Trans. on Antennas and Propagation, 2005.

[18] M. Dorigo, M. Birattari, and T. Stitzle, Ant Colony Optimization: Arificial Ants as a Computational Intelligence Technique, IEEE computational intelligence magazine, November, 2006.

[19] Leguizamon, G., Michalewicz, Z.: A new version of ant system for subset problems. In: Evolutionary Computation, 1999. CEC 99. Proceedings of the 1999 Congress on. Volume 2. 1999

[20] Fidanova, S.: Aco algorithm for mkp using different heuristic information. Lecture Notes in Computer Science 2542, pp.438-444, 2003

[21] Alaya, I., Solnon, C., Gh`edira, K.: Ant algorithm for the multi-dimensional knapsack problem. In: International Conference on Bioinspired Optimization Methods and their Applications (BIOMA 2004) pp.63-72, 2004. 
[22] Ji, J., Huang, Z., Liu, C., Liu, X., Zhong, N.: An Ant Colony Optimization Algorithm for Solving the Multidimensional Knap-sack Problems. In: Proceedings of the 2007 IEEE/WIC/ACM International Conference on Intelligent Agent Technology, IEEE Computer Society, pp.10-16, 2007.

[23] Shahrear Iqbal, Md. Faizul Bari, Dr. M. Sohel Rahman, A novel ACO technique for Fast and Near Optimal Solutions for the Multi-dimensional Multi-choice Knapsack Problem, Proceedings of 13th International Conference on Computer and Information Technology (ICCIT 2010), Dhaka, Bangladesh, pp.33-38, 2010.

[24] Stutzle, T., Hoos, H.H.: Max-min ant system. Future Generation Computer Systems 16, pp.889914, 2000.

[25] Skorin-Kapov, L., Mosmondor, M., Dobrijevic, O., Matijasevic, M.: Application-level QoS Negotiation and Signaling for Advanced Multimedia Services in the IMS. IEEE Comm. Magazine 45(7), pp.108-116, 2007.

[26] Dac-Nhuong Le, Optimizing Resource Allocation to Support QoS Requirements in Next Generation Networks using ACO Algorithm, International Journal of Computer Science and Information Technology \& Security (IJCSITS), Vol.2, No.5, pp.931-938, 2012.

[27] Dac-Nhuong Le, Optimizing the cMTS to Improve Quality of Service in Next Generation Networks based on ACO Algorithm, International Journal of Computer Network and Information Security (IJCNIS), Vol.5, No.4, 2013

[28] Dac-Nhuong Le, PSO and ACO Algorithms Applied to optimal Resource Allocation to Support QoS Requirements in Next Generation Networks, International Journal of Information \& Network Security (IJINS), Vol.2, No.3, pp.216-228, 2013.

[29] Dac-Nhuong Le, PSO and ACO Algorithms Applied to Optimizing Location of Controllers in Wireless Networks, International Journal of Computer Science and Telecommunications, Vol.3 No.10, pp.1-7.

[30] Dac-Nhuong Le, Nhu Gia Nguyen, and Vinh Trong Le, A Novel Ant Colony Optimization-based Algorithm for the Optimal Centralized Wireless Access Network, Lecture Notes of the Institute for Computer Sciences, Social Informatics and Telecommunications Engineering (LNICST), Springer 2013.

\section{Author's Profiles}

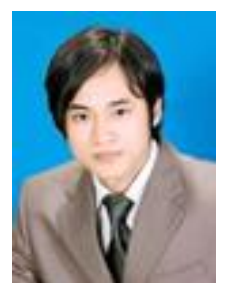

Dac-Nhuong Le received the BSc degree in computer science and the MSc degree in information technology from College of Technology, Vietnam National University, Vietnam, in 2005 and 2009 , respectively. He is a lecturer at the Faculty of information technology in Haiphong University, Vietnam. He is currently a Ph.D student at Hanoi University of Science, Vietnam National University. His research interests include algorithm theory, computer network and networks security.

How to cite this paper: Dac-Nhuong Le,"Optimizing QoS for Multimedia Services in Next Generation Network Based on ACO Algorithm", International Journal of Information Technology and Computer Science(IJITCS), vol.5, no.10, pp.30-38, 2013. DOI: $10.5815 /$ ijitcs.2013.10.04 